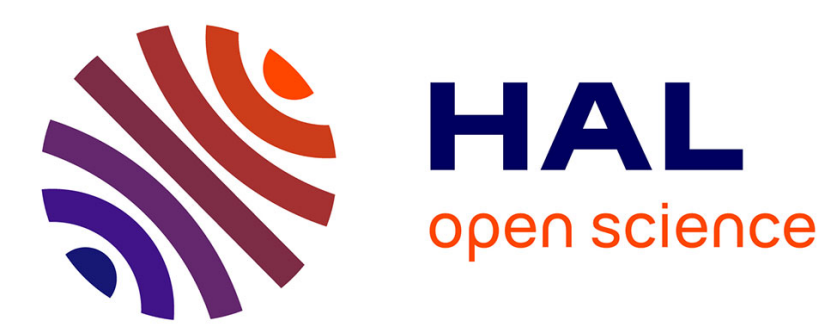

\title{
Entreprises privées et décision publique. Acteurs et organisation stratégique du lobbying dans les grandes entreprises en France \\ Madina Rival
}

\section{To cite this version:}

Madina Rival. Entreprises privées et décision publique. Acteurs et organisation stratégique du lobbying dans les grandes entreprises en France. Politiques et Management public, 2012, 29 (3), pp.431-449. 10.3166/pmp.29.431-449 . hal-02901099

\section{HAL Id: hal-02901099 \\ https://hal.science/hal-02901099}

Submitted on 17 Jul 2020

HAL is a multi-disciplinary open access archive for the deposit and dissemination of scientific research documents, whether they are published or not. The documents may come from teaching and research institutions in France or abroad, or from public or private research centers.
L'archive ouverte pluridisciplinaire HAL, est destinée au dépôt et à la diffusion de documents scientifiques de niveau recherche, publiés ou non, émanant des établissements d'enseignement et de recherche français ou étrangers, des laboratoires publics ou privés. 


\section{POLITIQUES \& \\ Politiques et management public}

Vol 29/3 | 2012

L'action publique en crise(s)?

\section{Entreprises privées et décision publique. Acteurs et organisation stratégique du lobbying dans les grandes entreprises en France}

Private firms and public decision: Players and places for lobbying in large firms in France

\section{Madina Rival}

\section{OpenEdition \\ Journals}

Édition électronique

URL : http://journals.openedition.org/pmp/5436

ISSN : $2119-4831$

Éditeur

Institut de Management Public (IDPM)

Édition imprimée

Date de publication : 15 septembre 2012

Pagination : 431-449

ISBN : 978-2-7430-1437-7

ISSN : 0758-1726

Ce document vous est offert par Conservatoire national des arts et métiers (Cnam)

\section{lecnam}

\section{Référence électronique}

Madina Rival, «Entreprises privées et décision publique. Acteurs et organisation stratégique du

lobbying dans les grandes entreprises en France », Politiques et management public [En ligne], Vol 29/3 2012, mis en ligne le 08 février 2015, consulté le 17 juillet 2020. URL : http://journals.openedition.org/ $\mathrm{pmp} / 5436$ 


\title{
Entreprises privées et décision publique Acteurs et organisation stratégique du lobbying dans les grandes entreprises en France
}

\author{
Madina Rival \\ Maître de conférences, LIRSA (Cnam) et LARGEPA- Université Paris 2 \\ 40 rue des jeûneurs -75002 Paris
}

\section{Résumé}

Suivant les temps et les lieux, les stratégies politiques des entreprises peuvent apparaître sous des vocables différents comme « lobbying ( (en anglais dans le texte), influence ou encore action politique (traduction du terme américain « Corporate Political Activity, CPA). Nous les définirons de manière générale comme toute activité consistant à procéder à des interventions destinées à modifier directement ou indirectement les processus d'élaboration, d'application ou d'interprétation de mesures législatives, normes, règlements et autres décisions des pouvoirs publics. Cet article souhaite contribuer à la compréhension des acteurs et de la fonction lobbying dans les grandes entreprises en France, dans le cadre d'une enquête réalisée par questionnaire. Le cadre théorique retenu est principalement celui des sciences de gestion.

(c) 2012 IDMP/Lavoisier SAS. Tous droits réservés

Mots clés : Lobbying, stratégies politiques d'entreprises, enquête.

\section{Abstract}

Private firms and public decision: Players and places for lobbying in large firms in France. At different times and places, corporate political strategies may appear under different terms such as «lobbying»(in English in the text), influence (or intelligence) or political (American translation of the term» Corporate Political Activity, CPA). We define broadly asany activity involving the conduct of interventions designed to directly or indirectly modify the development process, application or interpretation of legislation, standards, regulations and other policy decisions. This article aims to contribute to the description of players and places for lobbying in large firms in France with a

*Auteur correspondant : madina.rival@cnam.fr doi :10.3166/pmp.29.431-449 @ 2012 IDMP/Lavoisier SAS. Tous droits réservés 
questionnaire survey method. We will address this issue by mobilizing management literature to bring new light on this question.

(c) 2012 IDMP/Lavoisier SAS. Tous droits réservés

Keywords : Lobbying, corporate political strategies, inquiry.

\section{Introduction}

L'observation et l'étude de la stratégie sont devenues depuis les années 1970 l'un des fers de lance de la recherche et de l'enseignement en sciences de gestion. La conception traditionnelle de la stratégie reposait essentiellement sur une approche interne du dévelop-pement de l'entreprise ce qui ne correspond plus à la réalité du terrain. Aujourd'hui, les entreprises sont également (voire essentiellement) tournées vers leur environnement externe. Elles mettent en place des stratégies dites "hors marché », c'est-à-dire, non pas centrées sur leur produit mais sur l'influence des parties prenantes externes, dont la puissance publique.

Dans ce dernier cas, on parlera de stratégies politiques des entreprises. Suivant les temps et les lieux, elles peuvent apparaître sous des vocables différents comme «lobbying ( (en anglais dans le texte), influence (voire intelligence économique) ou encore action politique (traduction du terme américain « Corporate Political Activity », CPA). Nous les définirons de manière générale comme toute activité consistant à procéder à des interventions destinées à modifier directement ou indirectement les processus d'élaboration, d'application ou d'interprétation de mesures législatives, normes, règlements et autres décisions des pouvoirs publics.

«Booze, Blonds and Bribes »! Les stratégies politiques peuvent-elle se réduire à ces «trois $B$ » (de l'alcool, des blondes et des pots de vins) ? L'influence des intérêts privés sur la décision publique, puisque c'est de cela qu'il s'agit, fait couler beaucoup d'encre tout en étant souvent dissimulée dans les entreprises impliquées ! A contrario au sein des travaux existant en sciences de gestion, peu de données ont longtemps été disponibles sur les stratégies politiques des entreprises. Cet article souhaite contribuer à la compréhension de ces pratiques en répondant à la question suivante : quels sont aujourd'hui les acteurs et l'organisation stratégique du lobbying dans les grandes entreprises en France?

Nous y répondrons en mobilisant un corpus gestionnaire pour apporter un nouvel éclairage à cette question dans une première partie. La deuxième partie présente les écueils méthodologiques de l'étude du lobbying et la méthode d'enquête par questionnaire retenue ici. La troisième partie présente et discute les résultats en cherchant à conclure sur l'apport de notre recherche au management public.

\section{Comprendre le lobbying des entreprises : un défi pour les sciences de gestion}

Après avoir décrit comment les sciences de gestion (et en particulier le management stratégique) constituent un cadre propice pour comprendre cet objet (1.1), nous donnerons à voir les références qui nous ont permis de bâtir un questionnaire sur les acteurs et la fonction lobbying dans les entreprises françaises (1.2). 


\subsection{Une dimension politique à ancrer en management stratégique}

Une grande frustration à l'origine de nos recherches sur les stratégies politiques est le contraste saisissant entre le foisonnement des pratiques observées dans les entreprises et l'absence de théorisation en sciences de gestion. Pourtant, ce qui est peu banal en science de gestion, est largement connu dans d'autres disciplines.

En science politique, on distingue traditionnellement le groupe d'intérêt des autres groupes de la société (famille, tribu, ethnie par exemple) par son interaction - quelle qu'en soit la forme - avec les institutions de l'État et les partis engagés dans la lutte pour la conquête et l'exercice du pouvoir (Grossman et Saurugger, 2006). Les théories économiques du lobbying sont à rechercher dans le concept de "marché politique ». La théorie économique de la réglementation décrit l'utilisation par des groupes d'intérêt et des acteurs politiques des moyens de réglementation et du pouvoir coercitif des états pour orienter les lois et les règles dans des directions qui les favorisent (Stigler, 1975). De manière paradoxale, c'est également un économiste, Baron (1995), qui met en évidence l'existence d'un "non market environment ». "L'environnement hors marché » inclut les forces sociales, politiques et légales extérieures au marché mais en interaction avec les intérêts privés. Des stratégies hors marchés peuvent donc être définies, à savoir les stratégies politiques (lobbying notamment) destinées aux décideurs publics et les stratégies sociétales (responsabilité sociale de l'entreprise, désormais notée RSE) plus larges.

La sociologie enfin théorise de manière riche la notion d'influence, notamment au sein de réseaux sociaux dans lesquels les acteurs seraient encastrés. Nous pouvons tout d'abord, mobiliser la sociologie néo-institutionnelle pour préciser notre objet de recherche. Selon DiMaggio (1998) notamment, de nouvelles institutions apparaissent lorsque des acteurs organisés détenant des ressources suffisantes (les entrepreneurs institutionnels) y voient une opportunité de concrétiser des intérêts auxquels ils accordent de l'importance. On distingue traditionnellement deux parties dans l'activité d'entreprenariat institutionnel : la théorisation et la construction de coalitions qui pourrait s'apparenter à du lobbying dans le cas des associations que nous avons étudiées. Par la suite, le courant des effets sociétaux (notamment Sorge, 2005) peut permettre l'interprétation des différences entre les stratégies politiques des différents pays. Rival (2012) met à cet égard en balance les aspects globaux et locaux de plus de cinq cents observations.

Pour autant, nos recherches sur les stratégies politiques des entreprises sont ancrées en sciences de gestion et plus particulièrement en management stratégique. Cette thématique a une origine traditionnelle dans le marketing puisque qu'elle y est définie comme un mode de communication non traditionnel (ou hors médias) aux côtés des relations publiques, du parrainage ou du mécénat par exemple (Malaval et Décaudin, 2012). Néanmoins, l'essentiel de la littérature gestionnaire sur le lobbying provient du management stratégique. En 1962, Chandler définit la stratégie dans Strategy and Structure ${ }^{1}$ comme la détermination des buts et des objectifs de long terme d'une entreprise, l'adoption de moyens d'action et l'allocations des ressources nécessaires pour atteindre les objectifs chiffrés. Depuis une quinzaine d'années, force est de constater que cette vision de la stratégie d'une entreprise centrée sur

\footnotetext{
'A. Chandler (1962), Strategy and Structure: Chapters in the History of the American Industrial Enterprise, MIT Press, Cambridge.
} 
la production et la vente d'un bien ou d'un service est obsolète. Une part importante de la stratégie interne comme du conseil externe est aujourd'hui tournée vers l'environnement de l'entreprise. Ainsi, en partant du modèle des cinq forces proposé par Porter (1986), certains auteurs construisent un modèle des « $5+1$ » forces qui suppose une stratégie politique des entreprises tournée vers la puissance publique. De même, la notion de «partie prenante» développée à la suite de Freeman (1984) et qui intègre toute forme d'acteurs en lien avec l'entreprise élargit à l'État la réflexion sur l'espace stratégique pertinent.

\subsection{La description des stratégies de lobbying par le corpus des stratégies politiques des organisations}

En sciences de gestion, il existe peu de travaux académiques portant explicitement et uniquement sur les stratégies politiques des entreprises. Ce corpus dit des «Corporate Political Activities » (CPA) est une sous discipline reconnue du management stratégique tel qu'il est étudié et enseigné aux États-Unis ${ }^{2}$. Deux types de références peuvent être distingués en la matière : des analyses descriptives des différents éléments du lobbying (notamment acteurs et fonction) et des études plus normatives questionnant en particulier la performance des stratégies politiques. Pour les besoins de notre recherche, nous nous focaliserons principalement sur les articles descriptifs souvent constitués de typologies. L'ambition de cette partie n'est pas de recenser les travaux sur les stratégies politiques des entreprises mais simplement de donner les éléments de description des acteurs et de la fonction qui ont servi à l'élaboration du questionnaire donné en annexe de l'article.

\section{Les acteurs du lobbying}

L'auteur du lobbying peut être un cabinet extérieur (Lamarque, 1996) ou un agent interne à l'entreprise, unité spécialisée (Attarça, 1999) ou non en la matière. Cette problématique est classique en théorie des organisations : elle oppose l'internalisation à la sous-traitance des activités de lobbying. Chaque option présente des avantages et des inconvénients qu'il convient de peser. Ainsi, la création d'une structure spécifique au sein de l'entreprise évite les conflits d'intérêts (ou problèmes d'agence pour reprendre la terminologie de Jensen et Meckling (1976)) et permet une meilleure connaissance des intérêts à défendre alliée à la permanence des ressources. En revanche ce choix implique l'existence d'une taille et de moyens considérables, des difficultés d'organisation hiérarchique et un risque d'enclavement. À l'opposé, le recours à un cabinet de conseil permet l'accès à des lobbyistes d'expérience pour un risque plus limité mais offre le désavantage d'une défense d'intérêts plus tiède, voire de conflits d'intérêts et de problèmes de confidentialité d'informations stratégiques pour l'entreprise.

Ensuite, il peut s'agir d'une action individuelle ou collective (structurée ou non). Le choix entre ces deux options appartient à l'entreprise qui décide en fonction de la nature du sujet et de la cible mais aussi d'opportunités qui s'offrent à elle de rejoindre des groupes déjà formés. Elle raisonne en évaluant le rapport bénéfices/coûts. Les bénéfices à attendre d'une action collective sont de plusieurs ordres : poids supplémentaire dans

\footnotetext{
${ }^{2}$ L'Academy Of Management (AOM) a par exemple une division SIM (Social Issues In Management) qui draine l'ensemble des présentations sur les CPA.
} 
les négociations et crédibilité, économies d'échelle, efficacité, compétitivité, synergies, possibilité accrue de lutte contre des stratégies politiques individuelles opportunistes. Des coûts existent cependant et sont à prendre en compte : coût d'opportunité d'une action individuelle qui aurait pu être profitable à l'entreprise mais qui va à l'encontre des intérêts du groupe, voire ponctuellement d'une action contraire aux intérêts de la firme mais favorable à la majorité des lobbyistes réunis; coût de contrôle de l'activité des autres membres.

Getz (1993) dresse enfin une typologie de quarante-huit cibles potentielles en croisant quatre critères : l'origine de la cible (gouvernementale ou non), son niveau géographique (international, national, régional, local), sa fonction (exécutif, législatif, judiciaire), l'accès de l'entreprise à cette source (interne ou externe). Ces différentes cibles sont autant de choix de localisation et de répartition des lobbyistes à effectuer pour une entreprise en fonction de ses problématiques.

\section{L'organisation stratégique du lobbying}

D'après Miles (1978), de manière générale, une entreprise peut agir aussi bien en tant que : « prospecteur » (impliquant la nécessité d'une surveillance ex ante de l'environnement et donc d'une veille stratégique), « défenseur » (c'est à dire par une réaction ordonnée expost à une modification de l'environnement) ou « analyste » (action hybride). Une analyse concrète de cette problématique en lien avec le lobbying est proposée par Demil (1998). Elle concerne l'étude empirique du traitement des déchets dans le secteur hospitalier et aboutit à la conclusion qu'aucune des stratégies n'est la meilleure.

En outre, selon Farnel (1993) il existe deux options stratégiques fondamentales concernant les relations entre l'entreprise et les pouvoirs publics : la coopération « continue » ou l'approche « ad hoc». Une approche continue permettrait en externe l'établissement de relations de confiance et en interne un effet d'apprentissage pour les parties de l'entreprise concernées par l'action politique. En revanche, cette approche continue supposerait un investissement de ressources plus important de la part de l'entreprise qui doit être comparé avec les bénéfices retirés.

Le tableau dressé par Corrado (1984) permet d'opposer quelques techniques : mode de communication direct/indirect, mode d'influence relationnel/financier. Jacomet (2000) quant à lui a identifié deux types de stratégies de lobbying différentes : la " pression » et «l'interaction». La stratégie de pression reposerait sur un comportement de domination, voire de capture, de la part de l'entreprise sur les pouvoirs publics (et aussi sur les autres groupes d'intérêt). À l'opposé, la stratégie d'interaction privilégierait un mode de relation plus équilibré avec les pouvoirs publics.

Enfin, Attarça (1999) distingue les ressources informationnelles, relationnelles, symboliques, organisationnelles, économiques et financières dédiées au lobbying.

\section{Mesurer et observer le lobbying des entreprises en France : un défi pour le chercheur}

Découvrir ce qui est caché est la base de l'activité du chercheur. Elle est un défi de taille lorsque l'on s'intéresse aux stratégies politiques des organisations, plus particulièrement en France. C'est la raison pour laquelle la réflexion méthodologique est centrale sur notre objet de recherche. Nous la présenterons en 2.1 avant d'en venir à l'enquête menée sur les grandes entreprises françaises en 2010 en 2.2. 


\subsection{Les différentes approches méthodologiques du lobbying des entreprises}

La répartition entre approche qualitative et quantitative peut paraître quelque peu artificielle. Nous lui préférons celle liée au recueil des données qui peut se faire soit au niveau de la cible des stratégies politiques soit de l'organisation qui mène l'action politique.

L'approche par la cible génère l'intégralité des publications sur les « political strategies » dans des revues américaines de premier rang. Ces articles mettent en place des modèles explicatifs mathématiques de complexité inégale. Les variables chiffrées explicatives et expliquées sont des données publiques ayant trait au lobbying (dépenses, taille des lobbys, performance du lobbying par exemple). Elles sont disponibles au États-Unis auprès des organisations publiques cibles en raison d'une réglementation particulière de la vie politique. Ainsi, les campagnes sont financées par les entreprises au travers des PAC $^{3}$ qui se doivent d'être transparents sur leurs comptes. Par ailleurs la Disclosure law de 1995 rend obligatoire la transparence sur les sommes dépensées et leur destination en matière de lobbying. Enfin, un certain nombre d'autorités (proches des autorités administratives indépendantes françaises comme par exemple la Federal Communications Commission) disposent de données très précises sur les groupes d'intérêt et les sommes dépensées (De Figueiredo, Kim, 2004).

En France, il n'a pendant longtemps pas été possible de disposer de telles données. En effet le financement de la vie politique française est plus restrictif qu'aux États-Unis et exclusivement limité aux personnes physiques depuis les lois des années 1990. Le code pénal quant à lui condamne respectivement la corruption passive, la prise illégale d'intérêts et le trafic d'influence. Cependant, le statut des groupes d'intérêt change aujourd'hui en Europe et en France. Des registres mis en place très récemment (de 2008 à 2011) à la Commission et au Parlement Européen ainsi qu'au Sénat et à l'Assemblée Nationale en France permettront bientôt d'avoir davantage d'information.

Dans l'attente de ces éléments, une approche de l'entreprise lobbyiste est tout d'abord possible. Cette approche peut être externe. La presse permet notamment le recueil massif de données secondaires (Rival, 2012). Dans un second temps, une compréhension interne des stratégies politiques se révèle complémentaire. Elle peut passer par l'analyse de données secondaires fabriquées par l'organisation comme des documents comptables. Elle peut également prendre la forme du recueil de données primaires par l'administration (à distance) d'un questionnaire ou le recueil d'interviews. Ainsi, Tian et Deng (2007) traitent de manière statistique 201 questionnaires sensés définir les stratégies politiques des entreprises (en réalité administrés à des étudiants de MBA). C'est le choix que nous avons fait ici. Des études de cas peuvent enfin reposer sur des interviews au sein des organisations lobbyistes. Plus l'acteur est spécifique, plus ce type de démarche se justifiera (Lévy et Rival, 2010).

\subsection{Une enquête sur le lobbying des 120 plus grandes entreprises en France}

Une enquête a été conduite auprès des 120 plus grandes entreprises françaises et filiales d'entreprises étrangères installées en France (critère du chiffre d'affaires et du nombre de salariés) sous la forme d'un questionnaire. Ce document a été élaboré en collaboration avec une dizaine de professionnels des affaires publiques (lobbyistes d'entreprise, cabinets et personnel du Sénat et de l'Assemblée Nationale) afin de pouvoir comparer les réponses

${ }^{3}$ Political Action Committees. 
et en retirer des prescriptions opérationnelles. Le contenu du questionnaire se base sur la littérature descriptive des actions de lobbying évoquée dans la partie précédente. Il est notamment structuré autour de deux thématiques.

- les acteurs du lobbying: Cette partie s'interroge sur l'existence d'une structure de lobbying interne et son histoire; elle met en exergue notamment deux problématiques majeures tant sur le plan théorique que managérial : celle de l'externalisation de la fonction politique de l'entreprise et celle de sa gestion collective;

- la place du lobbying dans l'organisation et la stratégie de l'entreprise : L'idée générale de cette partie du questionnaire est de décrire le positionnement fonctionnel et stratégique pour une grande entreprise de ses relations avec le décideur public.

De manière transversale, ces deux parties sont traversées par la question des ressources (notamment humaines et financières mais aussi symboliques) du lobbying évoquées dans la littérature de la première partie.

Le questionnaire figurant en annexe de cet article a été administré avec plusieurs relances (mail et téléphone), à la fois par mail aux responsables affaires publiques et par courrier aux dirigeants des entreprises sélectionnées.

Entre mai et juillet 2010,23,3\% des sondés ont répondu à cette enquête 4 . Dans la suite de l'article, les entreprises ayant répondu à l'enquête seront considérées comme représentatives des 120 plus grandes entreprises françaises en raison du taux de réponse qui est statistiquement très bon. Par ailleurs, du point de vue de la taille, de la structure juridique ou financière et du secteur d'activité, ces 28 entreprises sont bien représentatives de l'échantillon initial (Tableaux 1 à 4).

L'échantillon contient de par sa construction de grandes entreprises essentiellement françaises (à l'exception d'une entreprise allemande). Les répondants sont majoritairement des sociétés anonymes cotées en bourse (Euronext), exerçant dans des secteurs comme les transports, la communication et l'énergie.

Les réponses à choix multiples de cette enquête ont été traitées sous la forme de statistiques descriptives sous SPSS. Des calculs d'effectifs (nombre d'entreprises ayant répondu positivement pour une modalité) et de pourcentages ont été effectués par modalité de réponse à chaque question. En cela une partie de cette étude est de nature quantitative. En revanche, les verbatims répondant aux questions plus ouvertes ont donné lieu à une interprétation qualitative. L'ensemble permet une meilleure vision de ce qui se pratique officiellement dans les grandes entreprises en France quant au rapport avec les décideurs publics.

Tableau 1 : Taille des entreprises

\begin{tabular}{|l|c|c|c|c|c|}
\hline Libellé & Effectif & Moyenne & Ecart-type & Minimum & Maximum \\
\hline CA 2009 (milliards d'euros) & 28 & 4818 & 24354 & 1 & 131327 \\
\hline RH 2009 (nombre de salariés) & 28 & 79211 & 80326 & 800 & 287000 \\
\hline
\end{tabular}

\footnotetext{
${ }^{4}$ Liste des 28 entreprises répondantes: Air France; Air Liquide; Auchan; Axa;BMW ; Eramet ; FranceTelecom (Orange) ; GRDF ; Groupe La Poste; Groupe Publicis; Iliad ; Imerys ; Lactalis ; Leroy Merlin ; Michelin ; Norbert Dentressangle; Pages Jaunes Groupe; Pernod Ricard; PSA; RATP; RFF; Rhodia; RTE; Schneider Electric; SNCF; Société générale ; Thales ; Total.
} 
Tableau 2 : Statut juridiques des entreprises

\begin{tabular}{|l|c|c|}
\hline & Effectifs & Pourcentages \\
\hline SA & 21 & 75,00 \\
\hline Filiale & 1 & 3,57 \\
\hline Société en nom collectif & 1 & 3,57 \\
\hline $\begin{array}{l}\text { Société en commandite } \\
\text { par actions }\end{array}$ & 1 & 3,57 \\
\hline Holding & 1 & 3,57 \\
\hline EPIC & 3 & 10,71 \\
\hline
\end{tabular}

Tableau 4 : Les entreprises cotées

\begin{tabular}{|l|c|c|}
\hline & Effectifs & Pourcentages \\
\hline oui & 19 & 67,86 \\
\hline non & 9 & 32,14 \\
\hline Ensemble & 28 & 100,00 \\
\hline
\end{tabular}

Tableau 3 : Secteur d'activité des entreprises

\begin{tabular}{|c|c|c|}
\hline & Effectifs & Pourcentages \\
\hline Transport & 5 & 17,86 \\
\hline Commerce & 2 & 7,14 \\
\hline Finance & 2 & 7,14 \\
\hline Industrie automobile & 3 & 10,71 \\
\hline Mines & 2 & 7,14 \\
\hline Communication & 5 & 17,86 \\
\hline Énergie & 5 & 17,86 \\
\hline Agroalimentaire & 2 & 7,14 \\
\hline Chimie & 1 & 3,57 \\
\hline Industrie & 1 & 3,57 \\
\hline
\end{tabular}

\section{Le lobbying : une réalité pour l'entreprise qui a de multiples prolongements}

Cette troisième partie de l'article présente les résultats de l'enquête (3.1) avant de les discuter et de les mettre en perspective (3.2).

\subsection{Les résultats de l'enquête : acteurs et fonction du lobbying au sein des grandes entreprises en France}

Il convient de noter avant toutes choses que les résultats que nous allons présenter sont à nuancer. En effet, ils ne concernent que 23,3\% de l'échantillon initial.

\section{Les acteurs du lobbying}

Seuls $7 \%$ des répondants affirment ne pas avoir d'équipe de lobbying. Pour reprendre la terminologie de Freeman (1984), l'État (ou plus largement la décision publique) est bien une «partie prenante » prise en compte par les grandes entreprises françaises. La localisation géographique de base est la France, doublée dans 50 \% des cas par un bureau à Bruxelles et/ou dans certains pays. Les pays évoqués sont : Pologne, Hongrie, Italie, Portugal, UK, Allemagne, USA, Japon, Italie, USA, Chine, Slovénie, Indonésie, Belgique, Espagne, Brésil, Russie, Thaïlande, Inde, Australie, Italie, l'Europe et les pays émergents en fonction des intérêts propres de chaque entreprise.

Une majorité d'entreprises privilégient un ensemble de personnes impliquées de manière coordonnée (46\%). Le lobbying est une pratique qui progresse mais qui n'a pas encore gagné ses lettres de noblesse puisque seuls $17 \%$ des répondants déclarent avoir une équipe dédiée (Tableaux 5 et 6 ). 
La question du profil du responsable de l'équipe de lobbying (Tableau 7) s'est avérée peu discriminante, avec une légère préférence pour les managers internes qui maîtrisent peut-être mieux l'aspect technique des dossiers à défendre. Ils distancent légèrement les profils politiques (32\%) et juridiques (28\%). La réponse à la question du titre du responsable est relativement homogène sur deux registres : Directeur (chef) des affaires publiques (relations institutionnelles et éventuellement européennes)/Conseiller de titre divers ou membre du cabinet du président... le mot lobbying n'est jamais utilisé.

Près de la moitié des entreprises répondantes ont une équipe de lobbying depuis plus de 5 ans contre $35 \%$ depuis moins de 5 ans (Tableau 10). La pratique n'est finalement pas si neuve. En revanche, elle n'est plus en évolution : aucun des répondants ne déclare avoir prévu de créer un poste de lobbyiste.

De manière un peu surprenante, près d'un tiers des entreprises interrogées a choisi de ne pas externaliser leur lobbying (Tableau 9). Pour près d'un autre tiers ce sera un cabinet spécialisé qui sera choisi, de préférence à un cabinet d'avocat ou une agence de communication.

Une majorité écrasante d'entreprises (92\%) traitent également leur lobbying au travers des organisations professionnelles sectorielles, au niveau français comme au niveau européen (Tableau 8). On peut citer par exemple la FFSA (Fédération Française des Sociétés d'Assurance) ou encore l'AFOM (Association Française des Opérateurs Mobiles). Près de la moitié a également recours à des organisations professionnelles généralistes (de type MEDEF) et simplement $17 \%$ s'adressent à des groupes ad hoc. Les entreprises sont pour moitié en responsabilité dans ces groupes, impliquées à $35 \%$ mais rarement créateurs $(3 \%)$. Une telle responsabilité donne du pouvoir mais crée également des obligations pour les entreprises en question... Le lobbying collectif revêt une forte importance pour plus de la moitié des répondants (c'est certainement la raison pour laquelle ils sont en responsabilité dans ces syndicats professionnels). Il est peut-être jugé plus efficace que le lobbying individuel.

\section{Tableau 5 :}

Avez-vous une équipe de lobbying?

\begin{tabular}{|l|c|c|}
\hline & Effectifs & Pourcentages \\
\hline En France & 26 & 92,86 \\
\hline À Bruxelles & 14 & 50,00 \\
\hline Dans certains pays & 14 & 50,00 \\
\hline Dans chaque pays & 0 & 0,00 \\
\hline $\begin{array}{l}\text { Pas d'équipe } \\
\text { lobbying }\end{array}$ & 2 & 7,14 \\
\hline
\end{tabular}

Tableau 6 : L'équipe de lobbying interne est-elle composée de?

\begin{tabular}{|l|c|c|}
\hline & Effectifs & Pourcentages \\
\hline une équipe dédiée & 5 & 17,86 \\
\hline une personne seule & 3 & 10,71 \\
\hline $\begin{array}{l}\text { un ensemble } \\
\text { de personnes impliquées } \\
\text { de manière diffuse }\end{array}$ & 4 & 14,29 \\
\hline $\begin{array}{l}\text { un ensemble de personnes } \\
\text { impliquées de manière } \\
\text { coordonnée }\end{array}$ & 13 & 46,43 \\
\hline NSP & 3 & 10,71 \\
\hline
\end{tabular}


Tableau 7 : Quel est le profil du responsable de votre équipe?

\begin{tabular}{|l|c|c|}
\hline & Effectifs & Pourcentages \\
\hline Juridique & 8 & 28,57 \\
\hline Politique & 9 & 32,14 \\
\hline Communication & 4 & 14,29 \\
\hline Manager interne & 10 & 35,71 \\
\hline Autre & 5 & 17,86 \\
\hline
\end{tabular}

Tableau 9 : Le lobbying est-il également externalisé?

\begin{tabular}{|l|c|c|}
\hline & Effectifs & Pourcentages \\
\hline $\begin{array}{l}\text { Pas } \\
\text { d'externalisation }\end{array}$ & 8 & 28,57 \\
\hline $\begin{array}{l}\text { Un cabinet } \\
\text { de lobbying }\end{array}$ & 8 & 28,57 \\
\hline $\begin{array}{l}\text { Un cabinet } \\
\text { d'avocats }\end{array}$ & 3 & 10,71 \\
\hline $\begin{array}{l}\text { Une agence de } \\
\text { communication }\end{array}$ & 4 & 14,29 \\
\hline $\begin{array}{l}\text { Une autre } \\
\text { structure }\end{array}$ & 2 & 7,14 \\
\hline
\end{tabular}

Tableau 8 : Depuis quand existe-il un responsable lobbying dans l'entreprise?

\begin{tabular}{|l|c|c|}
\hline & Effectifs & Pourcentages \\
\hline $\begin{array}{l}\text { Organisations } \\
\text { professionnelles } \\
\text { sectorielles }\end{array}$ & 26 & 92,86 \\
\hline $\begin{array}{l}\text { Organisations } \\
\text { professionnelles } \\
\text { généralistes }\end{array}$ & 12 & 42,86 \\
\hline Groupes ad hoc & 5 & 17,86 \\
\hline Autres groupes & 0 & 0,00 \\
\hline
\end{tabular}

Tableau 10 : Le lobbying est-il réalisé de manière collective?

\begin{tabular}{|l|c|c|}
\hline & Effectifs & Pourcentages \\
\hline Un poste va être créé & 0 & 0 \\
\hline Depuis moins de 5 ans & 10 & 35,71 \\
\hline Depuis plus de 5 ans & 12 & 42,86 \\
\hline $\begin{array}{l}\text { La date de création n'est } \\
\text { pas identifiable }\end{array}$ & 3 & 10,71 \\
\hline NSP & 3 & 10,71 \\
\hline
\end{tabular}

\section{La place du lobbying dans l'organisation et la stratégie des entreprises}

Le lobbying est souvent proche de la direction générale : rattaché à la Direction Générale dans $71 \%$ des cas et à une direction fonctionnelle dans $25 \%$ (Tableau 11). Le rattachement à une direction géographique est plus rare et celui à une direction produit inexistant. La direction qui comprend le lobbying est dans un certain nombre de cas une direction multitâche qui va gérer dans le même temps : la communication, les relations de presse, la gestion de crise, les partenariats et le développement durable (Tableau 12). Elle est plus rarement en charge des questions juridiques. Au sujet de la ligne hiérarchique (Tableau 14), on observe également une Direction Générale très proche du lobbying (rattachement direct dans $60 \%$ des cas et séparation par au plus une fonction pour les autres entreprises).

Le responsable du lobbying siège ou participe au comité de direction du groupe dans $42 \%$ des cas (Tableau 14). Dans la même idée que les questions 9 et 11, on observe ici une proximité importante entre les lieux de pouvoir de l'entreprise et le lobbying.... sauf dans les $28 \%$ d'entreprises qui n'informent qu'ex post leur responsable lobbying des décisions du comité de direction du groupe. L'équipe de lobbyistes est présente (souvent 
informée à $35 \%$ ) mais pas majoritairement décisionnaire (sollicitée pour donner son avis dans seulement $28 \%$ des cas et rarement informée dans $21 \%$ (Tableau 15). Très majoritairement la stratégie de lobbying est définie pour l'ensemble de l'entreprise (stratégie « corporate » à $89 \%$ ) ce qui assure peut-être une meilleure cohérence d'ensemble. Cette stratégie d'ensemble cohabite dans un quart des cas avec une stratégie par métier et/ou une stratégie par filiale (Tableau 16).

Au final, la question financière est encore parfois taboue, $15 \%$ des répondants n'ont pas souhaité renseigner cet élément. La moitié des répondants se situent dans la fourchette basse de dépenses (moins de 500000 euros) mais $21 \%$ déclarent dépenser entre 1 et 5 millions d'euros par an hors effectif. La communication et la représentation sont traditionnellement les postes de dépense les plus importants, sauf pour les entreprises qui ont recours à des lobbyistes extérieurs.

Tableau 11 : Quel est le rattachement hiérarchique de vos équipes lobbying?

\begin{tabular}{|l|c|c|}
\hline & Effectifs & Pourcentages \\
\hline Direction générale & 20 & 71,43 \\
\hline $\begin{array}{l}\text { Direction } \\
\text { fonctionnelle }\end{array}$ & 7 & 25,00 \\
\hline $\begin{array}{l}\text { Direction } \\
\text { géographique }\end{array}$ & 3 & 10,71 \\
\hline Direction produit & 0 & 0,00 \\
\hline
\end{tabular}

Tableau 13 : Pouvez-vous décrire la ligne hiérarchique entre le lobbying et la direction?

\begin{tabular}{|l|c|c|}
\hline & Effectifs & Pourcentages \\
\hline $\begin{array}{l}\text { Directement } \\
\text { rattaché à la DG }\end{array}$ & 17 & 60,71 \\
\hline $\begin{array}{l}\text { Séparé par une } \\
\text { fonction des la DG }\end{array}$ & 8 & 28,57 \\
\hline $\begin{array}{l}\text { Séparé par deux } \\
\text { fonctions de la DG }\end{array}$ & 0 & 0,00 \\
\hline $\begin{array}{l}\text { Très éloigné de } \\
\text { la DG }\end{array}$ & 0 & 0,00 \\
\hline NSP & 3 & 10,71 \\
\hline
\end{tabular}

Tableau 12 : Quelles sont les autres composantes que gère votre direction?

\begin{tabular}{|l|c|c|}
\hline & Effectifs & Pourcentages \\
\hline Communication & 8 & 28,57 \\
\hline Relation presse & 6 & 21,43 \\
\hline Gestion de crise & 8 & 28,57 \\
\hline Partenariats & 8 & 28,57 \\
\hline $\begin{array}{l}\text { Développement } \\
\text { durable }\end{array}$ & 9 & 32,14 \\
\hline Juridique & 3 & 10,71 \\
\hline Autre & 7 & 25,00 \\
\hline
\end{tabular}

Tableau 14 : Le responsable du lobbying participe-t-il au comité de direction du groupe?

\begin{tabular}{|l|c|c|}
\hline & Effectifs & Pourcentages \\
\hline Siège & 9 & 32,14 \\
\hline Participe & 3 & 10,71 \\
\hline $\begin{array}{l}\text { Participe } \\
\text { ponctuellement }\end{array}$ & 5 & 17,86 \\
\hline $\begin{array}{l}\text { N'est informé } \\
\text { qu'ex post }\end{array}$ & 8 & 28,57 \\
\hline NSP & 3 & 10,71 \\
\hline
\end{tabular}


Tableau 15 : Quelle est l'implication de l'équipe de lobbying dans les décisions majeures du groupe?

\begin{tabular}{|l|c|c|}
\hline & Effectifs & Pourcentages \\
\hline $\begin{array}{l}\text { Sollicitée pour donner } \\
\text { son avis }\end{array}$ & 8 & 28,57 \\
\hline Souvent informée & 10 & 35,71 \\
\hline Rarement informée & 6 & 21,43 \\
\hline Jamais informée & 2 & 7,14 \\
\hline NSP & 2 & 7,14 \\
\hline
\end{tabular}

Tableau 16 : Définition de la stratégie de lobbying et de communication

\begin{tabular}{|l|c|c|}
\hline & Effectifs & Pourcentages \\
\hline Corporate & 25 & 89,29 \\
\hline Par métier & 7 & 25,00 \\
\hline Par produit & 2 & 7,14 \\
\hline Par filiale & 7 & 25,00 \\
\hline
\end{tabular}

\section{2. Entreprises privées et décision publique : choix stratégiques privés et enjeux publics}

Notre interprétation des résultats de cette enquête est axée sur deux types d'enjeux : internes à l'entreprise sur ses choix stratégiques en matière de lobbying et externes pour les enseignements que le management public peut en retirer.

\section{Les choix stratégiques de l'entreprise lobbyiste}

Les choix stratégiques que nous avons pu observer dans le cas des opérations de lobbying sont finalement assez proches de ceux qui se posent dans le cadre de stratégies plus classiques. Ils peuvent être regroupés autour de trois questions : Faut-il internationaliser? Faut-il externaliser? Faut-il faire alliance au sein d'un groupement collectif?

À l'issue de cette étude, nous constatons que la localisation géographique pour la fonction «affaires publiques » est toujours la France, doublée dans $50 \%$ des cas par un bureau à Bruxelles et/ou dans certains pays. Les autres destinations évoquées sont des pays européens ou émergents en fonction des intérêts propres de chaque entreprise ${ }^{5}$.

Le deuxième type de choix stratégique oppose l'internalisation à la sous-traitance des activités de lobbying. Chaque option présente des avantages et des inconvénients qu'il convient de peser. Ainsi, la création d'une structure spécifique au sein de l'entreprise évite les conflits d'intérêts et permet une meilleure connaissance des intérêts à défendre alliée à la permanence des ressources. En revanche ce choix implique l'existence d'une taille et de moyens considérables, des difficultés d'organisation hiérarchique et un risque d'enclavement. À l'opposé, le recours à un cabinet de conseil permet l'accès à des lobbyistes d'expérience pour un risque plus limité mais offre le désavantage d'une défense d'intérêts plus tiède, voire de conflits d'intérêts et de problèmes de confidentialité d'informations stratégiques pour l'entreprise. Dans cet article, nous observons que l'internalisation est une pratique qui progresse mais qui n'a pas encore gagné ses lettres de noblesse puisque près de la moitié des entreprises répondantes ont une équipe de lobbying depuis plus de cinq ans contre $35 \%$ depuis moins de cinq ans. Près d'un tiers des entreprises interrogées a choisi

\footnotetext{
5 Pologne, Hongrie, Italie, Portugal, UK, Allemagne, USA, Japon, Italie, USA, Chine, Slovénie, Indonésie, Belgique, Espagne, Brésil, Russie, Thaïlande, Inde, Australie, Italie.
} 
de ne pas externaliser leur lobbying. Pour près d'un autre tiers ce sera un cabinet spécialisé qui sera choisi, de préférence à un cabinet d'avocat ou une agence de communication. La tendance à l'internalisation mise en évidence ici doit être nuancée par les spécificités de l'échantillon : nous avons étudié de très grosses entreprises dont les ressources humaines et financières rendent possibles de telles structures.

Enfin, l'action politique peut être menée de manière individuelle ou collective. Le choix entre ces deux options appartient à l'entreprise qui décide en fonction de la nature du sujet et de la cible mais aussi d'opportunités qui s'offrent à elle de rejoindre des groupes déjà formés. Les bénéfices à attendre d'une action collective sont de plusieurs ordres : poids supplémentaire dans les négociations et crédibilité, économies d'échelle, efficacité, compétitivité, synergies, possibilité accrue de lutte contre des stratégies politiques individuelles opportunistes. Des coûts existent cependant et sont à prendre en compte : coût d'opportunité d'une action individuelle qui aurait pu être profitable à l'entreprise mais qui va à l'encontre des intérêts du groupe ; coût de contrôle de l'activité des autres membres.

Notre enquête met en évidence qu'une majorité écrasante des grandes entreprises françaises $(92 \%)$ traitent leur lobbying au travers des organisations professionnelles sectorielles, au niveau français comme au niveau européen. En cela nous trouvons matérialisée la notion de construction de coalition définie par DiMaggio (1988) comme l'une des étapes clés de l'entrepreneuriat institutionnel. Près de la moitié a également recours à des organisations professionnelles généralistes (de type Medef $^{6}$ ) et simplement $17 \%$ s'adressent à des groupes ad hoc. Les entreprises sont pour moitié en responsabilité dans ces groupes, impliquées à $35 \%$ mais rarement à l'initiative $(3 \%)$. Une telle responsabilité donne du pouvoir mais crée des obligations pour les entreprises en question... Les stratégies collectives revêtent une forte importance pour plus de la moitié des répondants. Elles sont peut-être jugées plus efficaces que les stratégies individuelles. Notre constat converge sur ce point avec les travaux en sciences politiques : « $82 \%$ des associations listées par la Commission appartiennent aux organisations professionnelles des entreprises ou des syndicats » (Eising in Saurugger, 2003 : 160).

\section{Des enseignements pour le management public}

«Le management public correspond à l'ensemble des processus de finalisation, d'organisation, d'animation et de contrôle des organisations publiques visant à développer leur performance générale et à piloter leur évolution dans le respect de leur vocation. Il s'appuie sur un principe général du management qui est celui de la contingence : en d'autres termes il suppose une adaptation des méthodes de gestion à la diversité des situations et des enjeux (Bartoli, 2009 : 99-100). Un des éléments forts de cette contingence aujourd'hui est l'influence que les entreprises (et notamment les grosses structures) cherchent à exercer sur la décision publique. À la frontière entre le public et le privé, l'analyse des stratégies politiques des entreprises permet de repenser le management public. Cette discipline passionnante est elle-même en cours de structuration et nous avons la volonté d'y apporter notre contribution au-delà de l'ancrage en gestion revendiqué dans la première partie. La revue Politiques et Management Public nous semble être le lieu privilégié de cette réflexion.

Les politiques publiques sont concernées par nos travaux à double titre : le lobbying est un élément des politiques d'intelligence économique et concourt de manière plus

${ }^{6}$ Mouvement des entreprises de France. 
générale à la formation d'un nombre croissant de décisions publiques. De fait, l'influence est l'un des trois piliers de l'intelligence économique avec la veille et la sécurisation de l'information (Bournois et Romani, 2000). Elle a pour objet de renforcer la compétitivité d'un État, d'une entreprise ou d'un établissement de recherche. De manière plus générale, de multiples processus de décision publique (lois nationales ou directives européennes le plus souvent) portent l'empreinte de stratégies politiques. Cette remarque porte en elle une proposition de prolongement pour notre enquête : reprendre les 28 entreprises répondantes et étudier un ou des cas de processus de lobbying de manière longitudinale.

\section{Implications managériales et conclusion}

Le rapport de Jean-Marc Sauvé remis au Président de la République le 26 janvier 2011 et qui annonce une loi à venir sur les conflits d'intérêts ne consacre que très peu de place au lobbying. Seules deux pages sur les cent vingt et unes que comporte ce rapport s'intéressent en effet à la « représentation d'intérêts ». Elles décrivent le système existant en France et préconisent l'adoption des recommandations de bonnes pratiques dans des « codes de conduite et chartes de déontologie ». Il est vrai que le code pénal condamne déjà la corruption et le trafic d'influence tandis que de nombreuses lois réglementent le financement de la vie publique depuis 1988 et que des dispositions strictes encadrent déjà la commande publique. Pourquoi, dès lors, une telle passion médiatique pour le lobbying, souvent confondu avec la problématique des conflits d'intérêts? Peu de données sont facilement et publiquement disponibles sur le sujet du lobbying en France, que ce soit du côté des entreprises ou des pouvoirs publics.

L'enquête conduite ici auprès des 120 plus grandes entreprises françaises et filiales d'entreprises étrangères en France (critère du chiffre d'affaires) répond à cette attente. Les résultats mettent en lumière le paradoxe français. Le lobbying est une réalité structurante de la vie de ces entreprises : $93 \%$ disent avoir une équipe de lobbying (dont $50 \%$ depuis plus de 5 ans) qui est rattachée à la Direction Générale dans les trois quarts des cas... et pourtant les moyens mobilisés restent généralement limités puisque $50 \%$ des répondants y consacreraient moins de 500000 euros par an. De manière un peu surprenante, près d'un tiers des entreprises interrogées a choisi de ne pas externaliser leur lobbying. Pour près d'un autre tiers ce sera un cabinet spécialisé qui sera choisi, de préférence à un cabinet d'avocat ou une agence de communication. Une majorité écrasante d'entreprises (92\%) traitent également leur lobbying au travers des organisations professionnelles sectorielles, au niveau français comme au niveau européen. Près de la moitié a également recours à des organisations professionnelles généralistes (de type Medef). Au final, cette étude met en évidence à la fois des enjeux stratégiques internes à l'entreprise et externe en matière de management public.

Cette enquête présente néanmoins des limites, notamment sur le plan méthodologique. En effet, elle ne prend en compte que ce qui est dit officiellement par les entreprises interrogées. Il serait possible de croiser ces données avec celles des registres européens ou de l'Assemblée Nationale et du Sénat qui existent depuis deux ans mais nous serions encore dans l'univers du déclaratif. La piste de recherche la plus pertinente nous semble donc être celles d'une étude plus qualitative chez quelques individus de l'échantillon où la réalité du lobbying pourrait être perçue notamment au travers d'interviews d'acteurs du terrain (Lévy, Rival, 2010). 
Par ailleurs, d'autres problématiques liées à l'interaction public/privé auraient pu être abordées comme par exemple celle de l'éthique. À cet égard, certaines entreprises (comme par exemple Lafarge) mettent aujourd'hui en avant l'existence d'une charte de lobbying. Vigeo, l'agence de notation extra-financière, inclut même depuis quelques mois « la transparence et l'intégrité des stratégies et des pratiques d'influence » dans son référentiel de notation de la responsabilité sociale des entreprises.

\section{Remerciements}

Cet article a bénéficié du soutien de l'Association des Sciences Po. Néanmoins, les propos tenus n'engagent que l'auteur.

L'auteur tient à remercier les réviseurs de PMP pour la qualité et l'apport de leurs remarques.

\section{Bibliographie}

ATTARÇA M., (1999). Une introduction au concept de «stratégie politique d'entreprise », Thèse de doctorat de gestion, École des Hautes études Commerciales de Paris.

BARON D.P., (1995). Business and its Environment, Prentice Hall, Upper Saddle River.

BARTOLI A., (2009). Le management dans les organisations publiques, Dunod, Paris, 3 édition.

BOURNOIS F., Romani J.J., (2004). L’intelligence économique et comptable dans les entreprises françaises, Economica, Paris.

CORRADO F., (1984). Media for Manager, Prentice Hall, Upper Saddle River.

CHANDLER A., (1962). Strategy and Structure: Chapters in the History of the American Industrial Enterprise, MIT Press, Cambridge.

DAHAN N., (2005). Can There Be a Resource-Based View of Politics? International Studies of Management and Organizations 35 (2), 8-22.

DE FIGUEIREDO J., KIM J.(2004). When do Firm Hire Lobbyists? The Organization of Lobbying at the Federal Communications Commission, Industrial and Corporate Change, 13 (6), 883-900.

DEMIL B., (1998). Stratégie de pionnier et de suiveur : une application à un processus réglementaire, Thèse de doctorat de gestion, Université de Paris X Nanterre.

DIMAGGIO P.J., (1988). Interest and Agency in Institutional Theory In L.G. Zucker, Institutional Patterns and Organizations: Culture and Environment, Ballinger, Cambridge, 3-21.

EISING R. (2001). Associative Democracy in the European Union? Paper presented for the ECSA Seventh Biennial Conference, Madison, 31 mai-2 juin.

FARNEL F.J., (1993). Lelobbying : stratégies et techniques d'intervention, Éditions d'organisation, Paris.

FREEMAN R.E., (1984). Strategic Management, a Stakeholder Approach, Pitman, Boston.

GETZ K.A., (1993). Selecting Corporate Political Tactics In B. Mitnick (editor). Corporate Political Agency, Sage, Newbury Park, 242-273.

GROSSMAN E., Saurugger S., (2006). Les groupes d'intérêt : action collective et stratégies de représentation, Armand Colin, Paris.

JACOMET D., (2000). Les stratégies d'entreprise face aux politiques publiques : le lobbying des producteurs occidentaux et la politique commerciale internationale dans le textile habillement, Thèse de doctorat de gestion, Université Paris IX Dauphine. 
JENSEN M.C., Meckling W.H., (1976). Theory of the Firm, Managerial Behaviour, Agency Cost and Ownership Structure, Journal of Financial Economics 3 (4), 305-360.

LAMARQUE G., (1996). Le lobbying, Presses Universitaires de France, Paris.

LÉVY A., RIVAL M., (2010), Des formes d'entreprenariat dans les associations? Le cas des associations du secteurmédico-socialsoustutellepublique, Politiques et Management Public 27 ( 3), 55-72.

MALAVAL P., DÉCAUDIN J.M., (2012). Pentacom, Communication corporate, interne, financière, marketing $B$-to- $C$ et $B$-to- $B$, Pearson, Paris.

MILES R.E., (1978). Organizational Strategy: Structure and Process, McGraw Hill College Div., New York.

PORTER M., (1986). L'avantage concurrentiel, InterÉditions, Paris.

RIVAL M., (2012). Are Firms' Lobbying Strategies Universal? Comparison of Lobbying by French and UK Firms, Journal of Strategy and Management 5 ( 2), 211-230.

SAURUGGER S., (2003). Les groupes d'intérêts entre démocratie associative et mécanismes de contrôle, Raisons politiques 10 (2), 151-169.

SAUVÉ J.-M., (2011). Pour une nouvelle déontologie de la vie publique, Rapport de la Commission de réflexion pour la prévention des conflits d'intérêts dans la vie publique, remis au Président de la République le 26 janvier, Paris.

SORGE A., (2005). The Global and the Local, Oxford University Press, Oxford.

STIGLER G., (1975). The Citizen and the State: Essays on Regulation, University of Chicago Press, Chicago.

TIAN Z., DENG X., (2007). The Determinants of Corporate Political Strategy in Chinese Transition, Journal of Public Affairs 7, 341-356. 


\section{Annexe : Le questionnaire sur l'organisation du lobbying}

1. Avez-vous une équipe lobbying? :
a. $\square$ En France
b. $\square$ À Bruxelles
c. $\square$ Dans certains pays où vous êtes présents (lesquels)
d. $\square$ Dans chaque pays où vous êtes présents
e. $\square$ II n'y a pas d'équipe lobbying

En cas de réponse négative, répondre uniquement aux questions 4, 5,6, 7 et 8.

2. L'équipe de lobbying interne est-elle composée? :
a. $\square$ D'une personne seule
b. $\square D^{\prime}$ une équipe dédiée
c. $\square$ D'un ensemble de personnes impliquées de manière diffuse dans l'entreprise
d. $\square$ D'un ensemble de personnes impliquées de manière coordonnée dans l'entreprise

Précisez, si possible, le nombre de personnes concernées

3. Quel est le profil du responsable de votre équipe?
a. $\square$ Juridique
b. $\square$ Politique
c. $\square$ Communication
d. $\square$ Manager interne
e. $\square$ Autre

Quel est le titre de ce responsable?

4. Depuis quand existe-il un responsable du lobbying dans votre entreprise?
a. $\square$ Un poste va être créé
b. $\square$ Depuis moins de 5 ans
c. $\square$ Depuis plus de 5 ans
d. $\square$ La date de création n'est pas identifiable

5. Le lobbying est-il (également) externalisé?
a. $\square$ À un cabinet de lobbying
b. $\square A ̀$ un cabinet d'avocats
c. $\square A ̀$ une agence de communication ou de relations publiques
d. $\square A ̀$ une autre structure (précisez)

5bis Veuillez préciser les caractéristiques de la structure qui gère votre lobbying

6. Le lobbying est-il réalisé également de manière collective?
a. $\square$ Au sein d'organisations professionnelles sectorielles
b. $\square$ Au sein d'organisations professionnelles généralistes
c. $\square$ Au sein de groupes ad hoc
d. $\square$ Au sein d'autres groupes

6 bis Pouvez-vous préciser les noms de ces organisations? 
7. Quelle est votre implication dans cette organisation?
a. $\square$ Créateur du groupe
b. $\square$ En responsabilité dans le groupe
c. $\square$ Impliqué dans les actions du groupe
d. $\square$ Peu impliqué dans les actions du groupe

8. Quelle est l'importance du lobbying effectué par ces organisations pour votre groupe?
a. $\square$ Négligeable
b. $\square$ Moyenne
c. $\square$ Forte
d. $\square$ Très forte

9. Quel est le rattachement hiérarchique de vos équipes lobbying?
a. $\square$ Direction générale
b. $\square$ Direction fonctionnelle
c. $\square$ Direction géographique
d. $\square$ Direction produit

$\mathbf{9}$ bis Précisez si possible les intitulés de ces directions

10. Quelles sont les autres composantes que gère votre direction?
a. $\square$ Communication
b. $\square$ Relation presse
c. $\square$ Gestion de crise
d. $\square$ Partenariats (ONG, public, privé)
e. $\square$ Développement durable, RSE
f. $\square$ Juridique
g. $\square$ Autre

11. Pouvez-vous décrire en particulier la ligne hiérarchique entre le lobbying et la direction?
a. $\square$ Directement rattaché à la direction générale
b. $\square$ Séparé par une fonction de la direction générale
c. $\square$ Séparé par deux fonctions de la direction générale
d. $\square$ Très éloigné de la direction générale

12. Le responsable du lobbying participe il au comité de direction du groupe?
a. $\square$ Siège
b. $\square$ Participe
c. $\square$ Participe ponctuellement
d. $\square$ N'est informé qu'ex post

13. Concernant l'implication de l'équipe lobbying dans les décisions majeures du groupe (type plan marketing, lancement de produits)?, est-elle :
a. $\square$ Sollicitée pour donner son avis
b. $\square$ Souvent informée
c. $\square$ Rarement informée
d. $\square$ Jamais informée 
14. Définition de la stratégie de lobbying et de communication : s'agit-il d'une stratégie? :
a. $\square$ Corporate
b. $\square$ Par métier
c. $\square$ Par produit
d. $\square$ Par filiales

15. Quel budget hors effectif est alloué aux actions de lobbying dans l'entreprise en millions d'euros?
a. $\square$ Moins de 500000 euros
b. $\square$ Entre 500000 et 1 millions d'euros
c. $\square$ Entre 1 et 5 millions d'euros
d. $\square$ Plus de 5 millions d'euros
15 bis Précisez la ventilation de ce budget :
a. $\square$ Communication
b. $\square$ Abonnement à des bases de données
c. $\square$ Représentation
d. $\square$ Recours à des lobbyistes extérieurs

16. Observations/commentaires 
